\title{
Nacimiento y desarrollo de la Bioética
}

\author{
The birth and development of Bioethics
}

\section{José María Maya}

1. Médico, magíster en Salud Pública y en Dirección Universitaria; profesor titular de Ética y Salud Pública; ex rector, Universidad CES, Medellín, Colombia.

\section{RESUMEN}

Se presenta una síntesis de los antecedentes, el nacimiento y el desarrollo de la Bioética como disciplina contemporánea. Se busca llevar al lector desde el entorno en que surge la utilización del término 'bioética' en el mundo académico por Van Rensselaer Potter, hasta las discusiones actuales que propugnan el regreso al término 'ética' como respuesta a la necesidad de contar con una disciplina que sea aceptada por los denominados extraños morales en sociedades tan disímiles y pluralistas como las del mundo actual. Se hace una somera descripción de las principales tendencias de la bioética contemporánea para concluir que podemos interpretar a la bioética hoy, como un 'grito' en favor de la dignidad humana y de la convivencia pacífica y, a la vez, de la utilización de la ciencia y la tecnología en favor de una vida de calidad.

PALABRAS CLAVE: bioética, nacimiento, desarrollo histórico, tendencias.

\section{SUMMARY}

This article presents a summary of the birth, development and background of bioethics as contemporary discipline. It seeks to lead the reader from the arena in which the term "bioethics" emerges in the scholarly world by Van Rensselaer Potter to current discussions, advocating a return to the term 'ethics' in response to the need to have a discipline that is accepted by the so called moralist strangers in such diverse and pluralistic societies as today's world has. A brief description of the main trends in contemporary bioethics is presented. The article concludes that bioethics can be understood as a 'cry' on behalf of human dignity and peaceful coexistence and, at the same time, science and technology use on behalf of a quality life.

KEYWORDS: Bioethics, birth, trends, historic development.

\section{INTRODUCCIÓN}

Históricamente se ha planteado que el término 'bioética' fue introducido en el mundo académico en 1970 por el bioquímico y oncólogo estadounidense Van Rensselaer Potter de la University of Wisconsin, en un artículo publicado en la revista científica Perspectives in Biology and Medicine titulado "Bioethics: The science of survival"1. Un año más tarde, Potter publicó el libro "Bioethics: A bridge to the future" ${ }^{2}$, generando un interesante movimiento en el mundo académico, tanto alrededor del término como de los alcances y las perspectivas de la 'nueva disciplina'.

\author{
Correspondencia: \\ José María Maya \\ Email: \\ josemariamayamejia@gmail.com \\ Recibido: 25 de enero de 2016 \\ Aceptado: 3 de febrero de 2016 \\ No se reportan conflictos de \\ interés.
}




\title{
“[...] piensen la bioética como una nueva ética científica que combina la humildad, la responsabilidad y la competencia, que es interdisciplinaria e intercultural y que intensifica el sentido de la humanidad [...]"3.
}

\author{
Van Rensselaer Potter
}

Con este término, Potter aludía a los problemas que el gran desarrollo de la tecnología plantea a un mundo en plena crisis de valores. Urgía así a superar la ruptura entre la ciencia y la tecnología, por una parte, y las humanidades, por la otra. Esta fisura hunde sus raíces en la asimetría existente entre el enorme desarrollo tecnológico que otorga al ser humano el poder de manipular su intimidad y alterar el medio, y la ausencia de un aumento correlativo en su sentido de responsabilidad por el que habría de obligarse a sí mismo a orientar este nuevo poder en beneficio del propio ser humano y de su entorno natural.

El entorno en el que se genera esta corriente contemporánea de la ética, está marcado por una gran preocupación por el alcance de la investigación científica y la tendencia a dar preponderancia a la ciencia y sus posibilidades por encima de los seres humanos y sus derechos. Incluso, se revela en los prolegómenos del fenómeno, un temor sobre el futuro de la especie humana y la calidad de vida en el futuro, fundado en la realidad geopolítica internacional y en las tendencias a convertir la ciencia $\mathrm{y}$, por ende, la investigación científica, en parte de la lucha por la hegemonía de los sistemas políticos, y en la búsqueda del conocimiento científico como un fin en sí mismo, sin importar el efecto sobre los seres humanos participantes del proceso investigativo.

Para Potter, la bioética sería una nueva disciplina que tendería un lazo entre la ciencia y las humanidades o, con mayor precisión, un puente entre las ciencias biológicas y la ética. Para él, la supervivencia de la especie humana en una civilización decente y sustentable, requeriría del desarrollo y el mantenimiento de un sistema ético.

Potter, al final de su vida, sintetizó a la bioética como una nueva disciplina que combina el conocimiento y la reflexión en la búsqueda continua de la sabiduría, es decir, del conocimiento para la supervivencia humana y la mejoría de la condición humana en todo sentido. Concluye su reflexión pidiendo que:

“[...] piensen la bioética como una nueva ética científica que combina la humildad, la responsabilidad y la competencia, que es interdisciplinaria e intercultural y que intensifica el sentido de la humanidad [...]"3.

Esta definición es, además de su legado, el resultado de la maduración de su propuesta de los años 70, al avanzar hacia el concepto de "ética global” para superar la visión clínica, que fue el sesgo que esfuerzos como el de André Hellegers, fundador del Kennedy Institute of Ethics (también conocido como el Joseph and Rose Kennedy Institute of Ethics), introdujeron en el desarrollo de la bioética. En ella, Potter trata de unir la bioética clínica y la bioética ecológica, con el fin de poner en el centro de sus preocupaciones, los desafíos de la supervivencia del ser humano en el entorno de finales del siglo XX. Potter, preocupado por el curso seguido por su disciplina, terminó presentando una perspectiva globalizadora de la misma, dejando claro que no buscaba revivir la vieja ética médica ni reactivar una ética filosófica que no había podido dar cuenta de muchos de los problemas que aquejaban al ser humano contemporáneo. Este importante avance conceptual sitúa la bioética actual mucho más allá del horizonte dado por la definición, a partir de su etimología, de la Real Academia Española de la lengua y de las corrientes biomédicas contemporáneas.

\section{ANTECEDENTES Y TENDENCIAS}

Los fenómenos históricos, y entre ellos los nuevos movimientos académicos y sociales, no irrumpieron inesperadamente en el entorno, sino que fueron fruto de tendencias que se fueron incubando dentro de la his- 
toria de las colectividades y que en el mundo contemporáneo, dado su carácter de 'aldea global', tiene un impacto prácticamente planetario. Presentaremos algunos hitos históricos - sin pretender ser exhaustivos- de este proceso que condujo a la aparición y el desarrollo de la bioética como disciplina.

En 1949, Aldo Leopold, uno de los precursores del movimiento ecológico por la preservación ambiental, fue el primero en tratar de sustentar una relación entre la ética y la ecología, y esbozó la llamada ‘ética de la tierra' que, a finales del siglo XX y en estas primeras décadas del siglo $X X I$, ha vuelto a tomar auge, dentro del concepto de una bioética global y a tono con los movimientos como el de Albert Arnold Gore Jr., ex vicepresidente de los Estados Unidos, premio Nobel de la Paz, que manifiestan una gran preocupación por el futuro del planeta. Potter consideró la 'ética de la tierra' de Leopold como el principal antecedente y referente de la bioética ${ }^{4}$.

Dos años antes, en 1947, se había publicado el Código de Nuremberg como uno de los resultados del proceso del mismo nombre, en el cual se investigó, juzgó y condenó a muchos de los responsables de la cruel violación de los derechos humanos en los campos nazis de concentración. De ahí surge un valioso elemento, el "consentimiento informado" que irá irrumpiendo progresivamente en los procesos de investigación biomédica y, posteriormente, en la prestación de los servicios de salud.

En la década del 6o, Potter, haciendo eco de su preocupación por la calidad de vida en el futuro, creó en la University of Wisconsin un comité interdisciplinario cuyo tema central era el futuro de la especie humana. Potter, siguiendo a la destacada antropóloga estadounidense Margaret Mead, defendía que la Universidad tenía un importante papel en la generación y el apoyo a propuestas que garantizaran la supervivencia y la calidad de la vida humana en el futuro. Estas ideas tuvieron amplia divulgación al ser publicadas en 1970 por la prestigiosa revista Science ${ }^{5}$.

El 16 de junio de 1966, Henry K. Beecher, profesor de Anestesiología de Harvard University, publicó en el New England Journal of Medicine, el artículo "Ethics and clinical research" en el que afirmaba que, al menos, el 12\% de los artículos publicados en una importante revista biomédica, empleaban procedimientos reñidos con la ética ${ }^{6}$. En 1970, Beecher publicó el libro "Research and the individual: Human studies" reiterando sus planteamientos y dilemas.

En julio de 1971, André Hellegers, médico gineco-obstetra, contando con un subsidio del matrimonio Sargent Shriver y Eunice Kennedy, fundó en Georgetown University el primer instituto universitario de bioética, el Joseph and Rose Kennedy Institute of Ethics, conocido mundialmente como el Kennedy Institute of Ethics, que fue inaugurado el $1^{\circ}$ de octubre de 1971. Hellegers recogía así el término acuñado por Potter, 'bioética', pero restringido a las cuestiones de la ética médica, campo en el que se situaría a partir de entonces con mayor frecuencia.

"The Belmont Report" de la National Commission for the Protection of Human Subjects of Biomedical and Behavioral Research se publicó en 19797. El informe es el resultado de un largo proceso iniciado cuando salió en 1972 a la luz pública en los Estados Unidos, la realidad del estudio de la historia natural de la sífilis no tratada, realizado en la localidad de Tuskegee, Alabama. En este estudio, pacientes de raza negra con sífilis fueron dejados sin tratamiento, a pesar de la aparición de la penicilina en 1942, con el fin de describir la historia natural de la enfermedad. La reacción de la opinión pública llevó a que el Congreso de los Estados Unidos creara en 1974 la National Commission for the Protection of Human Subjects of Biomedical and Behavioral Research. Uno de los objetivos de la Comisión fue determinar los principios éticos básicos que deben regir la investigación biomédica y de comportamiento con sujetos humanos, y desarrollar las directrices que se deben seguir para garantizar que tal investigación se lleve a cabo de acuerdo con esos principios.

El Belmont Report intentó resumir los principios éticos básicos identificados por la Comisión en el curso de sus deliberaciones. Fue el resultado de un intenso período de discusiones llevadas a cabo en febrero de 1976 en el Centro de Conferencias Belmont en el Smithsonian Institution, complementado por deliberaciones mensuales de la Comisión que se realizaron a lo largo de un período de casi cuatro años. Su resultado fue una declaración de principios éticos básicos y directrices que ha tenido gran acogida entre los estudiosos de la bioética y entre los organismos que financian investigación biomédica y del comportamiento a nivel mundial.

El caso de Tuskegee no fue la única manifestación del atropello de los derechos humanos en la investigación científica ${ }^{8}$. La opinión pública estadounidense se sacudió y protestó por el estudio de Willowbrook ${ }^{9}$, escuela pública donde se realizaron investigaciones entre 1963 y 1966 , en niños con retraso mental que fueron intencionalmente inoculados, oralmente o por inyección, con el virus de la hepatitis. Luego, se controlaron los efectos de la gammaglobulina para combatir la enfermedad. Estos casos eran la punta del iceberg de un fenómeno que preocupaba a científicos y humanistas, quienes favorecían el desarrollo de la bioética, su estudio y su aplicación en diferentes países.

En 1979, Tom Beauchamp y Jones Childress, profesores de Georgetown University, publicaron el libro "Biome- 
dical ethics” ${ }^{10}$ que marcó un hito en el desarrollo de la disciplina. Beauchamp, profesor de Filosofía, investigador del Kennedy Institute of Ethics y miembro de la comisión redactora del informe Belmont, y Childress, teólogo y filósofo, han tenido un importante protagonismo en las comisiones y organismos que buscan rescatar y aplicar normas para regular la investigación biomédica y aplicar los principios éticos a la práctica biomédica. En este libro, los autores definieron los cuatro principios de la bioética: autonomía, no maleficencia, beneficencia y justicia, considerando que estos principios son prima facie, esto es, que vinculan siempre que no colisionen entre ellos, en cuyo caso habría que dar prioridad a uno $\mathrm{u}$ otro, dependiendo del caso.

En 1988, el eminente neurólogo y bioeticista, Peter Whitehouse de Ohio ${ }^{11}$, acuñó el término de bioética profunda con el fin de resaltar el carácter más holístico de la bioética. En el mismo año, el teólogo Hans Kung promulgó la necesidad de una ética global o universal para una política global y económica con la que todas las naciones y pueblos de las más variadas culturas y creencias, pudieran comprometerse. Igualmente, planteó que era un requisito necesario para la paz mundial.

El núcleo de la ética está centrado en el ser humano. Kung estaba convencido de que sin un consenso ético básico sobre determinados valores, normas y actitudes, resulta imposible una convivencia humana digna, tanto en pequeñas como en grandes sociedades; un consenso que solo es alcanzable mediante el diálogo y el mutuo reconocimiento y aprecio. La ética mundial debe partir de un principio tan básico como antiguo: "todo ser humano debe recibir un trato humano" ${ }^{12}$.

Finalmente, es conveniente destacar los aportes del filósofo y médico $\mathrm{H}$. Tristam Engelhardt ${ }^{13}$ quien, no solo amplió el horizonte de la bioética siguiendo el concepto de ética global de Potter, sino que enfatizó el concepto de bioética secular (ética civil para otros) como la única bioética posible en un mundo de 'extraños morales' que es el mundo contemporáneo, caracterizado por grados variables pero crecientes de secularización, mayor pluralismo (no solo moral, sino epistemológico e informativo) y una tendencia democrática igualmente creciente ${ }^{14}$. Esta tendencia, que algunos denominan 'ética civil', ha venido ganando espacio en las sociedades contemporáneas, buscando proporcionar una estructura mínima ${ }^{15}$ que dé cabida al pluralismo moral y vincule en acuerdos colectivos a extraños morales, por exiguos que sean sus vínculos. Es una bioética que cada vez tiene menos respuestas definitivas a problemas concretos, sino que acepta áreas de incertidumbre y busca resolver con autoridad ética las controversias entre extraños morales sin recurrir a la fuerza, permitiendo mantener un lenguaje ético civil mínimo destinado a resolver 'lo bueno' en una determinada sociedad y en determinado espacio temporal.

$\mathrm{Al}$ respecto, una importante bioeticista contemporánea, Victoria Camps, afirmó:

“[...] Para bien o para mal, la bioética ha empezado a desarrollarse en el marco cultural e ideológico de eso que ha venido a llamarse 'postmodernidad' un marco en el que convergen, por un lado, el desempeño y el escepticismo con respecto a los ideales de emancipación del proyecto ilustrado, que es el proyecto moderno. Por otro lado, el reconocimiento de una sociedad laica, plural y multicultural que tiene que hacer cuadrar el círculo de poder de acuerdo con distintas perspectivas éticas procedentes de culturas asimismo diversas [...]"16.

El movimiento bioético es muy dinámico y oscila entre posiciones que reducen la disciplina a los problemas biomédicos relacionados con la investigación científica, y posiciones que insisten en que la extensión de la bioética es inmensa y abarca desde problemas biomédicos y de atención en salud, hasta problemas ambientales, políticas públicas en asignación de recursos y de generación de espacios que permitan al ciudadano ejercer al máximo su libertad y autonomía respetando la diversidad y sin hacer daño a los demás. Por ello, en algunos espacios, se plantea el regreso al vocablo 'ética', para evitar el sesgo clínico y biomédico del neologismo 'bioética'.

Lo importante es tener claro que la ética o la bioética, que ha venido ganando terreno desde finales del siglo $\mathrm{XX}$, es una forma de articular principios de comportamiento y reflexión distintos de la tradicional ética filosófica en dos sentidos:

A diferencia de la reflexión derivada de un sistema filosófico o de una tradición de creencias, importa la deliberación basada en el diálogo entre distintos grupos, personas o intereses. El diálogo es el sustento legitimador de la práctica.

La bioética es hoy una forma de reflexión, es una empresa no solo interdisciplinaria, sino multidisciplinaria y transdisciplinaria, puesto que la tendencia apunta hacia la búsqueda de un diálogo en el cual coincidan tanto los cometidos como los contenidos y los contextos, y en el que son tan importantes los conceptos filosóficos y las creencias, como los datos de las ciencias y las influencias fácticas del poder político e institucional.

Estas dos características, el carácter dialógico del procedimiento y la naturaleza integradora e transdiciplinaria de los contenidos, alejan a la bioética actual de una simple aplicación normativa de principios o reglas y de un conjunto de admoniciones o prohibiciones sobre cómo comportarse. 
De lo que sí podríamos hablar es que en la bioética contemporánea hay diferentes tendencias o corrientes, entre las que me parece importante destacar someramente las siguientes:

Bió́tica personalista. Parte de la dignidad como valor propio de la persona la cual se manifiesta en su capacidad intrínseca de realizar los valores. El fundamento de las orientaciones que debe apoyar las propuestas de solución a los dilemas éticos se encuentra en la naturaleza humana, ya que la persona es el centro.

Bioética consensual o civil. En esta corriente se busca establecer un mínimo de principios aplicables dentro de una sociedad pluralista y secular. Se basa en los postulados de la llamada 'ética de mínimos' que propone un conjunto de normas que deben cumplir todos los miembros de la sociedad y que sería producto del consenso social en torno a ciertos parámetros mínimos de convivencia.

BioÉticA CLínicA. Esta escuela sigue el enfoque biomédico que se da a partir de Hellegers con su trabajo en el Kennedy Institute of Ethics, y su centro de atención son los problemas suscitados por el avance en el conocimiento científico y tecnológico de su aplicación en los diferentes ámbitos de la salud humana.

Bió́tica Social. Su centro de interés es la problemática de la justicia y el desarrollo social como el mejoramiento de las condiciones de vida de la población en equilibrio con el medio en que interactúa. Hay un énfasis en la promoción de los derechos humanos en una sociedad plural.

Bió́tica Como CIENCIA de LA ComplejidAd. Esta corriente es menos conocida y surge desde la perspectiva de las ciencias de la complejidad. Entre los sistemas dinámicos de los que se ocupa la complejidad como ciencia, está la vida, la cual es analizada por la bioética que tiene carácter interdisciplinario y transdisciplinario, no lineal. Este estudio abarca, no solo la vida humana, sino las demás existentes y su interacción con los sistemas dinámicos.

Estas tendencias son comprensibles dados sus puntos de partida epistemológicos e históricos, pero no debe hacernos perder el hilo conductor de esta nueva disciplina y su carácter, entendiendo que son aproximaciones al mismo objeto de estudio y a una práctica social cada vez más centrada en el ser humano y sus relaciones con su naturaleza, los otros seres humanos y la organización social, y con los seres vivos en general y el universo.

Podemos concluir que la bioética es un grito a favor de la dignidad humana, que se materializa en las diferentes aplicaciones de los principios éticos. Es una forma de ser y de actuar que prioriza en todo momento y lugar la dignidad humana y el respeto a la vida, constituyéndose en una importante opción para la sociedad del siglo XXI.

\section{REFERENCIAS}

1. Potter VR. Bioethics, the science of survival. Perspect Biol Med. 1970;14:127-53.

2. Potter VR. Bioethics, bridge to the future. Englehood Cliffs, N.J.: Prentice Hall; 1971.

3. Potter VR. Bioética puente, bioética global y bioética profunda. En: Cuadernos del Programa Regional de Bioética. 1998;7:21-35.

4. Potter VR. Global bioethics. Building on the Leopold legacy. East Lansing, MI: Michigan State University Press; 1988.

5. Spinsanti S. Bioética global o la sabiduría para sobrevivir. En: Cuadernos del Programa Regional de Bioética. 1998;7:7-19.

6. Beecher HK. Ethics and clinic research. N Engl J Med. 1966;274:11354-6o.

7. Informe Belmont. Fecha de consulta: 25 de enero de 2016. Disponible en: http://www.bioeticaweb.com/content/ view/1060/857/.

8. Brand AM. Racism and research: The case of the Tuskegee Syphiles Study. Hastind Center Report. 1978;8:21-9.

9. Krugman S. The Willowbrook hepatitis studies revisited: Ethical aspects. Rev Infect Dis. 1986;8:157-62.

10. Beauchamp TL, Childress JF. Principles of biomedical ethics. Fifth edition. New York: Oxford University Press; 2001.

11. Whitehouse PJ. Van Rensselaer Potter: An intellectual memoir. Camb Q Healthc Ethics. 2002;11:331-4.

12. Küng H. Proyecto de una ética mundial. Madrid: Editorial Trotta; 1996.

13. Engelhardt T. The foundations of bioethics. New York: Oxford University Press; 1996.

14. Vidal M. Bioética. Estudios de bioética racional. Madrid: Editorial Tecnos; 1989.

15. Cortina A. Ética mínima. Madrid: Editorial Tenos; 1992.

16. Camps V. Un marco ético para la bioética. En: Palacios M, coordinador. Bioética. Oviedo: Ediciones Nobel; 200o. p. 49. 